\title{
Epoxy Resin Removal Technique in Cross-section Cuts for SEM
}

\section{Guillermina Gonzalez-Mancera}

\section{National Autonomous University of Mexico (UNAM), Mexico, Distrito Federal, Mexico}

The ultra-structure of specimens is studied many times in cross-section cuts getting for Transmission Electron Microscopy (TEM). There are several techniques to get the thin sections and gain information about the specimen, for example, by focused ion beam (FIB) that is included in a Dual Beam SEM. Richard Wirth (2009) exhibit FIB/TEM/SEM as appropriate techniques to sample and subsequently define the structure state of specimens on the nanometer scale. In general, FIB gives a very thin TEM foil, but many times is a method that require of time to determine the specific work conditions for each kind of specimen. Other option to get cross sections is by ultramicrotomy, after these films can be studied by TEM or SEM. However, the epoxy resin in which the specimen is into, represents an obstacle to observe internal structure of the sample if it is analyzed by SEM, because, the resin can cover the surface of the specimen. In this study it is proposed a technique to remove this resin. With this purpose, it is studied the ultrastructure of the internal layer (exine) of the pollen grain of the allergenic species Taraxacum officinale (Dandelion) Asteraceae family. Cross-sections from pollen were getting by ultramicrotome, after, they were placed on glass coverslips, then epoxy resin was eliminated by immerse them in sodium ethoxide, next they were cover with a thin film of carbon and gold, and finally these were observed by SEM. These utilized procedures generated the following results from the pollen of the specie studied here: internal ultra-structure of its exine, and details about the wall stratification. There are some works about studies of pollen by TEM, that show similar results from the internal structure of pollen like in this work. However, TEM technique can represent to invest more time and more effort to get analogous information than SEM. This proposed method has the advantage to be easy and quick, also it allows to show the ultrastructure with 3D appearance (Fig. 1), that in TEM is not possible (Fig. 2). It is suggested like a complementary method to get evidence of the internal structure from different kinds of samples. Besides, chemical microanalysis by EDS can be realized more readily and in bigger areas than by TEM.

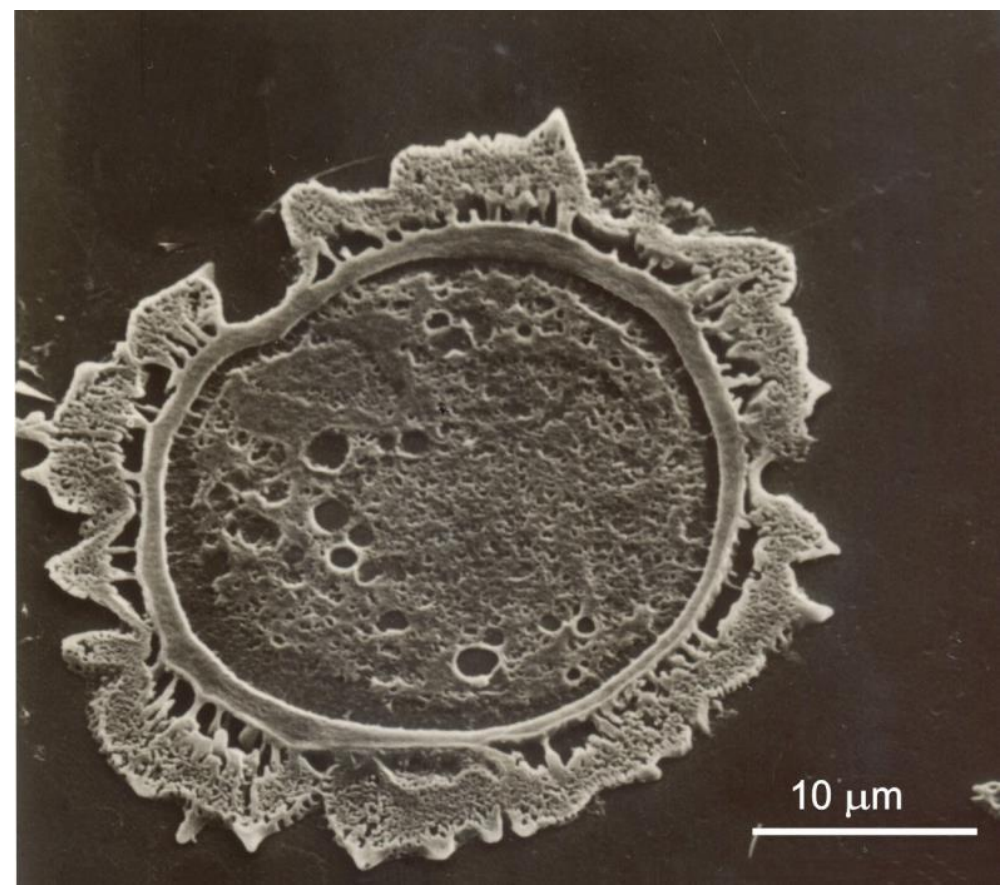

Figure 1. SEM image of cross-section of pollen of the T. officinale species 


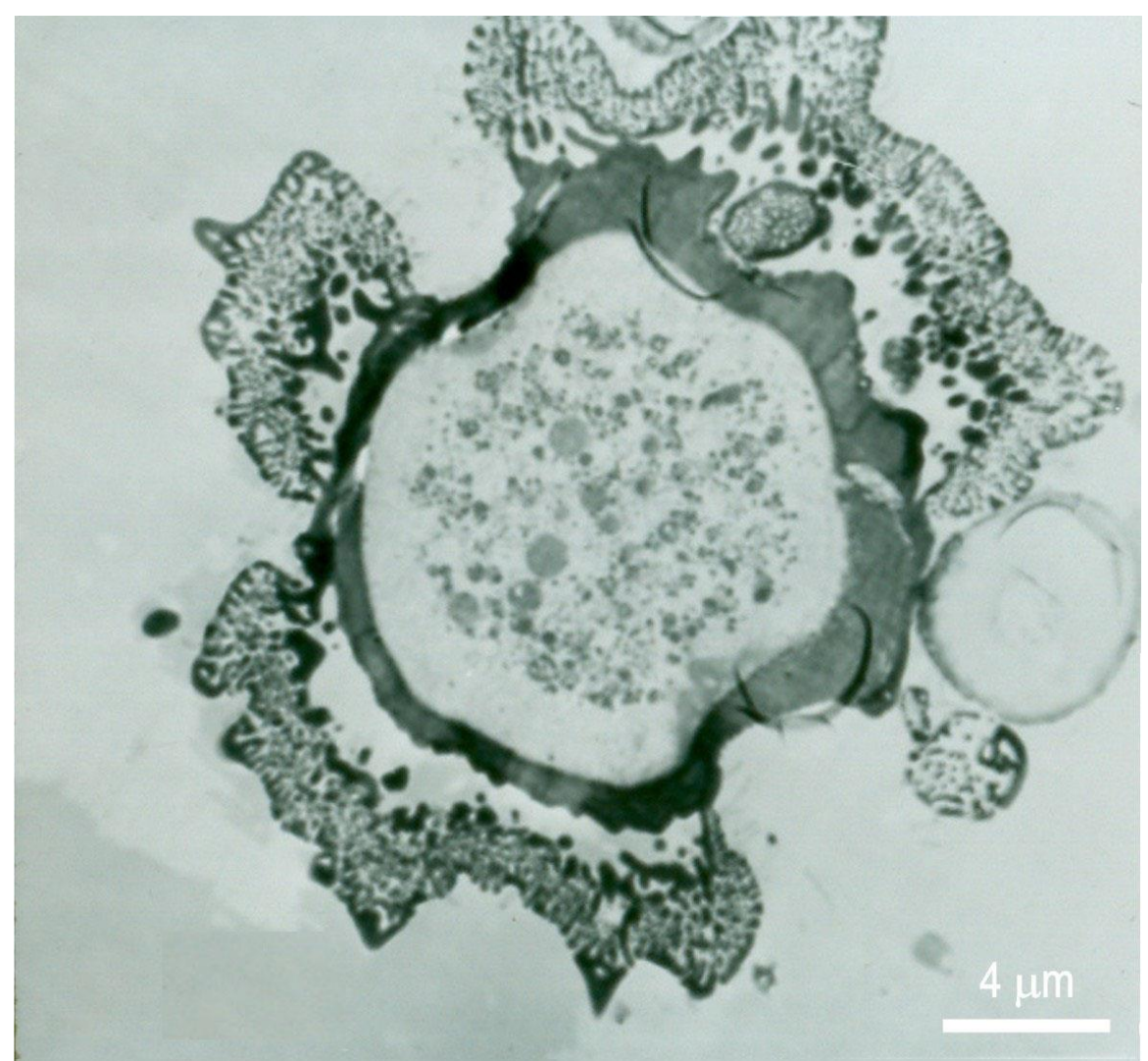

Figure 2. TEM image of cross-section of pollen of the T. officinale species

\section{References}

Wirth Richard, Focused Ion Beam (FIB) combined with SEM and TEM: Advanced Analytical Tools for Studies of Chemical Composition, Microstructure and Crystal structure in Geomaterials on a Nanometre scale. Chemical Geology, Elsevier. 2019, (261) 217-229. 